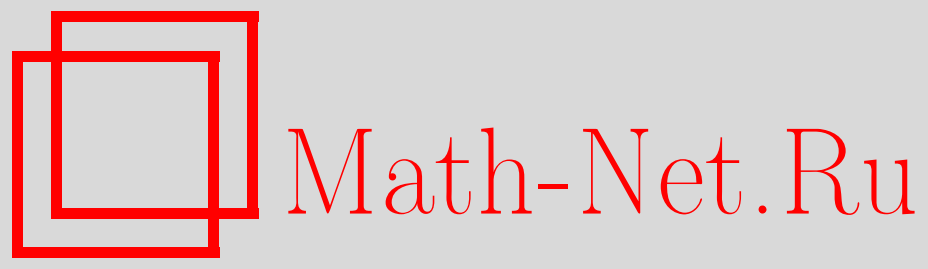

С. А. Назаров, Принудительная устойчивость простого собственного числа на непрерывном спектре волновода, Функи. анализ и его прил., 2013, том 47, выпуск 3, 37-53

DOI: https://doi.org/10.4213/faa3117

Использование Общероссийского математического портала MathNet.Ru подразумевает, что вы прочитали и согласны с пользовательским соглашением

http://www. mathnet.ru/rus/agreement

Параметры загрузки:

IP : 107.22 .136 .117

26 апреля 2023 г., 14:46:49

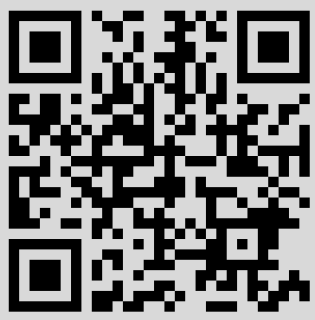




\title{
Принудительная устойчивость простого собственного числа на непрерывном спектре волновода*
}

\author{
(C) 2013. C. A. НАзАРов
}

\begin{abstract}
В статье показано, что при выполнении нескольких условий ортогональности и правильном выборе дополнительных параметров малое компактное возмущение оператора Гельмгольца не выводит из спектра простое собственное число, расположенное между порогами непрерывного спектра задачи Дирихле в области с цилиндрическим выходом на бесконечность. Результат получен посредством асимптотического анализа расширенной матрицы рассеяния.
\end{abstract}

\section{§1. Постановка задачи}

Пусть $\Omega$ - область в пространстве $\mathbb{R}^{d}$ с липшицевой границей $\partial \Omega$, совпадающая внутри полупространства $\mathbb{R}_{+}^{d}=\left\{x=(y, z): y=\left(x_{1}, \ldots, x_{d-1}\right) \in \mathbb{R}^{d-1}, z=\right.$ $\left.x_{d}>0\right\}$ с цилиндром $Q=\{x: y \in \omega, z \in \mathbb{R}\}$ (волноводом); здесь $\omega$ - область в $\mathbb{R}^{d-1}$ с границей $\partial \omega$ и компактным замыканием $\bar{\omega}=\omega \cup \partial \omega$. Считаем, что подмножество $G=\{x \in \Omega: z<0\}$ (резонатор) также имеет компактное замыкание $\bar{G}$. В области $\Omega$ рассмотрим спектральную задачу Дирихле для оператора Лапласа $\Delta$, а именно,

$$
-\Delta u(x)=\lambda u(x), \quad x \in \Omega, \quad u(x)=0, \quad x \in \partial \Omega .
$$

Вместе с задачей (1.1) изучаем возмущенную задачу, зависящую от малого параметра $\varepsilon>0$ и набора параметров $\boldsymbol{\tau}=\left(\tau_{0}, \tau_{1}, \ldots, \tau_{N}\right) \in \mathbb{R}^{1+N}$, где $\tau_{0}=1$, а остальные числа $\tau_{n}$ малые, порядка $\varepsilon$. В возмущенной спектральной задаче

$$
-\Delta u^{\varepsilon}(x)+\varepsilon L(\boldsymbol{\tau} ; x, \nabla) u^{\varepsilon}(x)=\lambda^{\varepsilon} u^{\varepsilon}(x), \quad x \in \Omega, \quad u^{\varepsilon}(x)=0, \quad x \in \partial \Omega,
$$

фигурирует формально самосопряженный дифференциальный оператор

$$
\begin{gathered}
L(\boldsymbol{\tau} ; x, \nabla)=\tau_{0} L_{0}(x, \nabla)+\cdots+\tau_{N} L_{N}(x, \nabla), \\
L_{n}(x, \nabla)=-\nabla^{\top} B_{n}(x) \nabla+\mathbf{b}_{n}(x)^{\top} \nabla-\nabla^{\top} \mathbf{b}_{n}(x)+b_{n}(x),
\end{gathered}
$$

где ${ }^{\top}$ - знак транспонирования, $\nabla$ - оператор-градиент, интерпретируемый как столбец, $B_{n}-$ симметричная $(d \times d)$-матрица, а $\mathbf{b}_{n}$ есть $d$-столбец, причем их элементы и свободный член $b_{n}$ - измеримые ограниченные вещественные функции с компактными носителями. Из-за возможных особенностей границы и коэффициентов обе задачи следует понимать как интегральные тождества

$$
\begin{gathered}
(\nabla u, \nabla v)_{\Omega}=\lambda(u, v)_{\Omega}, \quad v \in H_{0}^{1}(\Omega), \\
\left(\nabla u^{\varepsilon}, \nabla v^{\varepsilon}\right)_{\Omega}+\varepsilon l\left(\boldsymbol{\tau} ; u^{\varepsilon}, v^{\varepsilon}\right)=\lambda^{\varepsilon}\left(u^{\varepsilon}, v^{\varepsilon}\right)_{\Omega}, \quad v^{\varepsilon} \in H_{0}^{1}(\Omega) .
\end{gathered}
$$

\footnotetext{
* Работа выполнена при финансовой поддержке РФФИ (проект 12-01-00348).
} 
При этом $(\cdot, \cdot)_{\Omega}-$ скалярное произведение в пространстве Лебега $L_{2}(\Omega)$, а $H_{0}^{1}(\Omega)$ - пространство Соболева функций, обращающихся в нуль на $\partial \Omega$. В интегральном тождестве (1.5) для задачи (1.2) появился дополнительный член

$$
\begin{gathered}
l=\tau_{0} l_{0}+\cdots+\tau_{N} l_{N}, \\
l_{n}(u, v)=\left(B_{n} \nabla u, \nabla v\right)_{\Omega}+\left(\mathbf{b}_{n}^{\top} \nabla u, v\right)_{\Omega}+\left(u, \mathbf{b}_{n}^{\top} \nabla v\right)_{\Omega}+\left(b_{n} u, v\right)_{\Omega} .
\end{gathered}
$$

Полуторалинейная форма в левой части формулы (1.4) замкнута и положительно определена, так как ввиду цилиндрического строения волновода $\{x \in Q$ : $z>0\}$ и ограниченности резонатора $G$ выполнено неравенство

$$
\left\|u ; L_{2}(\Omega)\right\| \leqslant c_{\omega, G}\left\|\nabla u ; L_{2}(\Omega)\right\|, \quad u \in H_{0}^{1}(\Omega) .
$$

Таким образом, вариационной задаче ставится [1, гл. 10] в соответствие неограниченный положительно определенный самосопряженный оператор $A$ в $L_{2}(\Omega)$. Точно так же возникает самосопряженный оператор $A^{\varepsilon}$, который остается положительно определенным при малом $\varepsilon$ благодаря неравенству (1.7) и оценкам

$$
\left|l_{n}(u, u)\right| \leqslant c_{n}\left(\left\|u ; L_{2}(\Omega)\right\|^{2}+\left\|\nabla u ; L_{2}(\Omega)\right\|^{2}\right), \quad n=0, \ldots, N,
$$

для форм (1.6). Возмущение происходит на компактном множестве, т. е. при условии, что $\varepsilon$ мало (дифференциальный оператор $\Delta_{x}-\varepsilon L$ должен сохранить эллиптичность), у операторов $A$ и $A^{\varepsilon}$ одинаковый непрерывный спектр $\sigma_{c}=\sigma_{c}^{\varepsilon}=\left[\Lambda_{1},+\infty\right)$, определяемый исключительно строением области $\Omega$ около бесконечности. Здесь $\Lambda_{1}$ - первое (простое и положительное) собственное число задачи

$$
-\Delta U(y)=\Lambda U(y), \quad y \in \omega, \quad U(y)=0, \quad y \in \partial \omega .
$$

Все ее собственные числа объединим в последовательность

$$
\Lambda_{1}<\Lambda_{2} \leqslant \Lambda_{3} \leqslant \cdots \leqslant \Lambda_{k} \leqslant \cdots \rightarrow+\infty,
$$

составленную с учетом кратностей, а отвечающие им (вещественные) собственные функции $U_{k} \in H_{0}^{1}(\omega)$ подчиним условиям ортогональности и нормировки

$$
\left(U_{j}, U_{k}\right)_{\omega}=\delta_{j, k}, \quad j, k \in \mathbb{N}:=\{1,2, \ldots\},
$$

где $\delta_{j, k}-$ символ Кронекера. Числа (1.9) образуют так называемые пороги и разбивают луч $\sigma_{c}=\sigma_{c}^{\varepsilon}$ на интервалы $\Upsilon_{p}=\left(\Lambda_{p}, \Lambda_{p+1}\right)$, непустые при $\Lambda_{p}<\Lambda_{p+1}$. Еще один интервал $\Upsilon_{0}=\left(0, \Lambda_{1}\right)$ включает дискретный спектр $\sigma_{d}$, а при малом $\varepsilon$ и возмущенный дискретный спектр $\sigma_{d}^{\varepsilon}$ оператора $A^{\varepsilon}$. Точечные спектры $\sigma_{p}$ и $\sigma_{p}^{\varepsilon}$ отличаются от дискретных собственными числами, вкрапленными в непрерывный спектр. Решения $u$ и $u^{\varepsilon}$ задач $(1.1)$ и $(1.2)$ из класса $H_{0}^{1}(\Omega)$ экспоненциально затухают на бесконечности и называются захваченными волнами.

Известно, что собственное число $\lambda \in \sigma_{d} \subset \Upsilon_{0}$ (с кратностью $\left.\varkappa\right)$ устойчиво, т. е. найдутся такие положительные величины $\varepsilon_{\lambda}$ и $C_{\lambda}, c_{\lambda}$, что при $\varepsilon \in\left(-\varepsilon_{\lambda}, \varepsilon_{\lambda}\right)$ в $C_{\lambda} \varepsilon$-окрестности точки $\lambda$ оператор $A^{\varepsilon}$ имеет в точности $\varkappa$ собственных чисел $\lambda_{q}^{\varepsilon} \in \sigma_{d}^{\varepsilon}$ и на интервале $\left(\lambda-c_{\lambda}, \lambda+c_{\lambda}\right)$ других собственных чисел нет. Собственные числа на непрерывном спектре не являются устойчивыми и при сколь угодно малом возмущении могут исчезать из спектра, превращаясь в точки комплексного резонанса (см. [2]-[4] и др.). Основная цель статьи - найти условия, 
при которых возмущение $\varepsilon L(\tau ; x, \nabla)$ оператора Лапласа не выводит из спектра простое собственное число, расположенное между порогами,

$$
\lambda \in\left(\Lambda_{N}, \Lambda_{N+1}\right) \quad\left(\Lambda_{N}<\Lambda_{N+1}\right) .
$$

Это делается при помощи анализа вспомогательного объекта - расширенной матрицы рассеяния $S^{\varepsilon}$, введенной в [5] для конических областей и в [6] для цилиндрических. Некоторый блок этой матрицы служит идентификатором точечного спектра (см. [6] и далее теорему 5). Появление одного и того же числа $N$ в формулах (1.11) и (1.3) не случайно: именно $N$ условий ортогональности (6.8), наложенных на «основное возмущение» $\varepsilon l_{0}$, и правильный подбор дополнительных малых параметров $\tau_{1}, \ldots, \tau_{N}=O(\varepsilon)$ («точная настройка» всей формы $\varepsilon l$ из (1.6) при учете требований (6.9)) обеспечивают сохранение собственного числа в непрерывном спектре. Вопрос о компенсации неустойчивости кратного собственного числа или группы простых остался открытым.

Задачу (1.2) можно получить из задачи (1.1) в области $\Omega(\varepsilon)$ с локально возмущенной границей путем спрямления последней. Соответствующие построения приведены в работах [7], [8], посвященных, в основном, исследованию асимптотики устойчивых собственных чисел из дискретного спектра.

\section{§2. Волны и операторы задач в весовых пространствах}

Обозначим через $W_{\beta}^{1}(\Omega)$ весовое пространство Соболева с показателем $\beta \in$ $\mathbb{R}$, которое состоит из функций $u \in H_{\mathrm{loc}}^{1}(\bar{\Omega})$, обладающих конечной нормой $\left\|u ; W_{\beta}^{1}(\Omega)\right\|=\left\|\exp (\beta z) u ; H^{1}(\Omega)\right\|$, а через $W_{\beta, 0}^{1}(\Omega)$ - подпространство функций, обращающихся в нуль на поверхности $\partial \Omega$ (оно может быть получено замыканием по той же норме линеала $C_{c}^{\infty}(\Omega)$ бесконечно дифференцируемых функций с компактными носителями). Зафиксируем параметр (1.11). В интегральном тождестве

$$
(\nabla u, \nabla v)_{\Omega}-\lambda(u, v)_{\Omega}=f(v), \quad v \in W_{-\beta, 0}^{1}(\Omega),
$$

под $f \in\left(W_{-\beta, 0}^{1}(\Omega)\right)^{*}$ понимается антилинейный непрерывный функционал на пространстве $W_{-\beta, 0}^{1}(\Omega),(\cdot, \cdot)_{\Omega}$ - расширение скалярного произведения в $L_{2}(\Omega)$ до двойственности между подходящими весовыми пространствами Лебега, a caмо решение $u$ ищется в классе $W_{\beta, 0}^{1}(\Omega)$. Задача $(2.1)$ порождает отображение

$$
W_{\beta, 0}^{1}(\Omega) \ni u \mapsto \mathscr{A}_{\beta} u=f \in\left(W_{-\beta, 0}^{1}(\Omega)\right)^{*},
$$

непрерывное при любом весовом показателе $\beta \in \mathbb{R}$. Другие свойства оператора $\mathscr{A}_{\beta}$, а также оператора $\mathscr{A}_{\beta}^{\varepsilon}$ (обозначение понятно) существенно зависят от $\beta$. По определению $\mathscr{A}_{\beta}$ и $\mathscr{A}_{-\beta}-$ взаимно сопряженные операторы.

В бесконечном цилиндре $Q$ существуют волны: осциллирующие

$$
w_{n}^{ \pm}(x)=\left(4\left(\lambda-\Lambda_{n}\right)\right)^{-1 / 4} \exp \left( \pm i z \sqrt{\lambda-\Lambda_{n}}\right) U_{n}(y), \quad n=1, \ldots, N,
$$

и экспоненциальные

$$
v_{j}^{ \pm}(x)=\left(4\left(\Lambda_{j}-\lambda\right)\right)^{-1 / 4} \exp \left( \pm z \sqrt{\Lambda_{j}-\lambda}\right) U_{j}(y), \quad j=N+1, N+2, \ldots .
$$

Каждая из них - решение однородной задачи Дирихле для уравнения Гельмгольца в цилиндре $Q$. После умножения на подходящую срезающую функцию 
$\chi \in C^{\infty}(\mathbb{R})$ волны $(2.2)$ попадают в пространство $W_{\beta, 0}^{1}(\Omega)$ при любом $\beta<0$, а волны $(2.3)-$ при $\beta<\mp \sqrt{\Lambda_{j}-\lambda}$. Удобно считать, что

$$
\chi(z)=1 \quad \text { при } z>\ell+1, \quad \chi(z)=0 \quad \text { при } z<\ell,
$$

причем $\chi=0$ на резонаторе $G$ и на носителях коэффициентов операторов (1.3).

Теория эллиптических задач в областях с цилиндрическими выходами на бесконечность (см., например, книгу [9, гл. 3,5$]$ и цитированную в ней литератору) устанавливает запретные весовые показатели $\beta$, при которых операторы $\mathscr{A}_{\beta}$ и $\mathscr{A}_{\beta}^{\varepsilon}$ теряют фредгольмовость. Эти показатели тесно связаны с показателями экспонент у волн (2.2) и (2.3). Сформулируем результаты, которые можно найти в гл. 5 из [9] или обзорах [10], [11], и отметим, что названная теория обычно имеет дело с краевыми задачами в классической постановке, но переход к обобщенным решениям не встречает сколь-нибудь заметных препятствий (ср. [11]).

Теорема 1. (1) Пусть $\lambda$ - число (1.11), а $\gamma$ удовлетворяет условию

$$
\gamma \in \Upsilon(\lambda):=\left(\sqrt{\Lambda_{J}-\lambda}, \sqrt{\Lambda_{J+1}-\lambda}\right) \quad\left(J>N, \Lambda_{J}<\Lambda_{J+1}\right) .
$$

Операторы $\mathscr{A}_{ \pm \gamma}$ фредгольмовы, и размерности их ядер связаны соотношениeм dim ker $\mathscr{A}_{-\gamma}=\operatorname{dim} \operatorname{ker} \mathscr{A}_{\gamma}+J . \Pi p u \gamma= \pm \sqrt{\Lambda_{J+1}-\lambda}$ uли $\gamma= \pm \sqrt{\Lambda_{J}-\lambda}$ операторы $\mathscr{A}_{ \pm \gamma}$ теряют фредгольмовость, так как их образы незамкнуты.

(2) Пусть $и \in W_{-\gamma, 0}^{1}(\Omega)$ - решение задачи (2.1) с весовым показателем $\beta=-\gamma$ u «быстро затухающей» правой частъю $f \in\left(W_{-\gamma, 0}^{1}(\Omega)\right)^{*}$ (последнее пространство включено в $\left.\left(W_{\gamma, 0}^{1}(\Omega)\right)^{*} \ni \mathscr{A}_{-\gamma} u\right)$. Тогда справедливо разложсение

$$
u(x)=\chi(x) \sum_{ \pm}\left(\sum_{n=1}^{N} a_{n}^{ \pm} w_{n}^{ \pm}(x)+\sum_{j=N+1}^{J} b_{j}^{ \pm} v_{j}^{ \pm}(x)\right)+\widetilde{u}(x),
$$

а коэфбициенты $a_{n}^{ \pm}, b_{j}^{ \pm}$и остаток $\widetilde{u} \in W_{\gamma, 0}^{1}(\Omega)$ подчинены неравенству

$$
\begin{aligned}
\left(\sum_{ \pm}\left(\sum_{n=1}^{N}\left|a_{n}^{ \pm}\right|^{2}+\sum_{j=N+1}^{J}\left|b_{j}^{ \pm}\right|^{2}\right)\right. & \left.+\left\|\widetilde{u} ; W_{\gamma}^{1}(\Omega)\right\|^{2}\right)^{1 / 2} \\
& \leqslant c_{\gamma}\left(\left\|f ;\left(W_{-\gamma, 0}^{1}(\Omega)\right)^{*}\right\|+\left\|u ; W_{-\gamma}^{1}(\Omega)\right\|\right) .
\end{aligned}
$$

Разложение (2.6) также может быть получено методом Фурье и обеспечивает формулу для размерностей ядер из теоремы 1, в которой последнее слагаемое есть не что иное, как половина количества волн, возникающих в разложении.

\section{§3. Пространства с отделенной асимптотикой и матрица рассеяния}

Пусть $\mathbf{W}_{\gamma}^{1}(\Omega)$ - прообраз подпространства $\left(W_{-\gamma, 0}^{1}(\Omega)\right)^{*}$ для оператора $\mathscr{A}_{-\gamma}$. По теореме 1 этот линеал состоит из функций $u$, допускающих представление $(2.6)$, и потому он назван [10] весовым классом с отделенной асимптотикой. Взяв левую часть формулы $(2.7)$ в качестве нормы $\left\|u ; \mathbf{W}_{\gamma}^{1}(\Omega)\right\|$, превращаем $\mathbf{W}_{\gamma}^{1}(\Omega)$ в гильбертово пространство. Сужение $\mathbf{A}_{\gamma}$ оператора $\mathscr{A}_{-\gamma}$ с $W_{-\gamma, 0}^{1}(\Omega)$ на $\mathbf{W}_{\gamma}^{1}(\Omega)$ наследует все свойства этого оператора, в частности, фредгольмовость и ядро с коядром. 
Зафиксируем весовой показатель $\beta \in\left(\sqrt{\lambda-\Lambda_{N}}, \sqrt{\Lambda_{N+1}-\lambda}\right)$. Теорема об асимптотике (см., например, [9, теорема 5.1.4]), аналогичная второму утверждению теоремы 1 , дает такие формулы для решения $u \in W_{-\beta, 0}^{1}(\Omega)$ задачи (2.1) с опять-таки «более быстро затухающей» правой частью $f \in\left(W_{-\beta, 0}^{1}(\Omega)\right)^{*}$ :

$$
\begin{gathered}
u(x)=\chi(x) \sum_{ \pm} \sum_{n=1}^{N} a_{n}^{ \pm} w_{n}^{ \pm}(x)+\widetilde{u}(x) \\
\left(\sum_{ \pm} \sum_{n=1}^{N}\left|a_{n}^{ \pm}\right|^{2}+\left\|\widetilde{u} ; W_{\beta}^{1}(\Omega)\right\|^{2}\right)^{1 / 2} \leqslant c_{\beta}\left(\left\|f ;\left(W_{-\beta, 0}^{1}(\Omega)\right)^{*}\right\|+\left\|u ; W_{-\beta}^{1}(\Omega)\right\|\right) .
\end{gathered}
$$

Подчеркнем, что из-за уменьшения весового показателя разложение (3.1) не содержит волны (2.3), а у самого решения $u$ нет экспоненциального роста.

В соответствии с волновым числом $\pm \sqrt{\lambda-\Lambda_{n}}$ осциллирующие волны $w_{n}^{+}$и $w_{n}^{-}$из (2.2) называются уходящими и приходящими соответственно. Дополним задачу (1.1) «физическими» условиями излучения и положим

$$
\left(\mathbf{W}_{\beta}^{1}(\Omega)\right)^{+}=\left\{u \in \mathbf{W}_{\beta}^{1}(\Omega): a_{n}^{-}=0, n=1, \ldots, N\right\} .
$$

Иными словами, в разложениях (3.1) функций из пространства (3.2) разрешены только уходящие волны. Очередное утверждение известно, но оно вытекает и из теоремы 3, доказываемой далее и обслуживающей более общую ситуацию.

Предложение 2. (1) B подпространстве $\operatorname{ker} \mathscr{A}_{-\beta} \ominus \operatorname{ker} \mathscr{A}_{\beta}$, имеющем размерность $N$, можно ввести базис $\zeta_{1}, \ldots, \zeta_{N}$, удовлетворяющий соотношения.M

$$
\zeta_{n}(x)=\chi(x) w_{n}^{-}(x)+\chi(x) \sum_{m=1}^{N} s_{m n} w_{m}^{+}(x)+\widetilde{\zeta}_{n}(x),
$$

где $\widetilde{\zeta}_{n} \in W_{\beta, 0}^{1}(\Omega)$, а $s=\left(s_{m n}\right)-$ унитарная $(N \times N)$-матрица рассеяния.

(2) У задачи (2.1) с правой частью $f \in\left(W_{-\beta, 0}^{1}(\Omega)\right)^{*}$ есть решение $u \in$ $\left(\mathbf{W}_{\beta}^{1}(\Omega)\right)^{+}$в том и только в том случае, когда $f(v)=0$ при всех $v \in \operatorname{ker} \mathscr{A}_{\beta}$. Это решение определено с точностью до слагаемого из подпространства $\operatorname{ker} \mathscr{A}_{\beta}$.

Аналогичные манипуляции с оператором $\mathscr{A}_{-\beta}^{\varepsilon}$ приводят к фредгольмову отображению $\mathbf{A}_{\beta}^{\varepsilon}: \mathbf{W}_{\beta}^{1}(\Omega) \rightarrow\left(W_{-\beta, 0}^{1}(\Omega)\right)^{*}$ и матрице рассеяния $s^{\varepsilon}$, причем предложение 2 сохраняет силу и для оператора возмущенной задачи (1.2).

\section{§4. Расширенная матрица рассеяния и ее считающее свойство}

Классификация [12] (см. также [9, §5.3]) волн (2.2) использует антиэрмитову форму

$$
\mathbf{q}_{R}(u, v)=\int_{\omega}\left(\overline{v(y, R)} \partial_{z} u(y, R)-u(y, R) \overline{\partial_{z} v(y, R)}\right) d y,
$$

которая возникает в формуле Грина как поверхностный интеграл и поэтому не зависит от параметра $R>\ell+1$ для решений $u$ и $v$ уравнения Гельмгольца в полуцилиндре $Q_{\ell}=\{x \in Q: z>\ell+1\}$, обращающихся в нуль на его боковой поверхности (в этом случае обозначаем форму символом q без индекса $R$ ). Согласно [12] и $[9, \S 5.3]$, волна $w-$ уходящая, если $\operatorname{Im} \mathbf{q}(w, w)>0$, и приходящая, 
если $\operatorname{Im} \mathbf{q}(w, w)<0$. В работе [6] были предложены к рассмотрению линейные комбинации (пакеты) экспоненциальных волн (2.3)

$$
w_{j}^{ \pm}(x)=2^{-1 / 2}\left(v_{j}^{+}(x) \mp i v_{j}^{-}(x)\right), \quad j=N+1, N+2, \ldots .
$$

Обе группы волн (2.2) и (4.2) удовлетворяют соотношениям

$$
\mathbf{q}\left(w_{j}^{ \pm}, w_{k}^{ \pm}\right)= \pm i \delta_{j, k}, \quad \mathbf{q}\left(w_{j}^{ \pm}, w_{k}^{\mp}\right)=0, \quad j, k=1, \ldots, J,
$$

обеспеченным, в частности, условиями ортогональности и нормировки (1.10). Поэтому и при $j=N+1, \ldots, J$ экспоненциальные волновые пакеты $w_{j}^{+}$и $w_{j}^{-}$ мы называем (условно) уходящими и приходящими соответственно.

Пусть $\lambda$ - собственное число оператора $A$ с кратностью $\varkappa$. Зафиксируем весовой показатель $(2.5)$ так, чтобы все соответствующие собственные функции $u_{1}, \ldots, u_{\varkappa} \in H_{0}^{1}(\Omega)$ не принадлежали $W_{\gamma, 0}^{1}(\Omega)$. Такой показатель $\gamma$ существует потому, что любое собственное число задачи (1.1) имеет конечную кратность (см. [9, замечание 3.1.5]) и не могут обратиться в нуль все коэффициенты ряда Фурье по затухающим волнам $(2.3)$ для функции $u_{k} \neq 0$ в полуцилиндре $Q_{\ell}$ (в противном случае $u_{k}=0$ всюду в $\Omega$ ). Тогда $\operatorname{dim} \operatorname{ker} \mathscr{A}_{\gamma}=0$ и $\operatorname{dim} \operatorname{ker} \mathscr{A}_{-\gamma}=$ $\operatorname{dim}$ coker $\mathscr{A}_{\gamma}=J$ по теореме 1. Равенством (3.2) с понятными изменениями введем подпространство $\left(\mathbf{W}_{\gamma}^{1}(\Omega)\right)^{+} \subset \mathbf{W}_{\gamma}^{1}(\Omega)$, разрешающее в отделенной асимптотике только уходящие волны $w_{1}^{+}, \ldots, w_{J}^{+}$. Сужение $\mathbf{A}_{\gamma}^{+}$оператора $\mathscr{A}_{-\gamma}$ на $\left(\mathbf{W}_{\gamma}^{1}(\Omega)\right)^{+}$- фредгольмов оператор. В следующем утверждении проверяем, что $\mathbf{A}_{\gamma}^{+}$- изоморфизм, а также определяем расширенную матрицу рассеяния $S$.

Теорема 3. (1) При любом $f \in\left(W_{-\gamma, 0}^{1}(\Omega)\right)^{*}$ задача (2.1) с $\beta=-\gamma$ имеет единственное решение $u \in\left(\mathbf{W}_{\gamma}^{1}(\Omega)\right)^{+}$, причем $\left\|u ;\left(\mathbf{W}_{\gamma}^{1}(\Omega)\right)^{+}\right\| \leqslant c\left\|f ;\left(W_{-\gamma, 0}^{1}(\Omega)\right)^{*}\right\|$.

(2) В ядре ker $\mathscr{A}_{-\gamma}$ существует базис $Z_{1}, \ldots, Z_{J} \in \mathbf{W}_{\gamma}^{1}(\Omega)$, для которого

$$
Z_{j}(x)=\chi(x) w_{j}^{-}(x)+\chi(x) \sum_{k=1}^{J} S_{k j} w_{k}^{+}(x)+\widetilde{Z}_{j}(x),
$$

где $\widetilde{Z}_{j} \in W_{\gamma, 0}^{1}(\Omega)$, а $S=\left(S_{k j}\right)-$ унитарная $(J \times J)$-матрица.

Доказательство. Первое утверждение вытекает из второго, так как

$$
\text { Ind } \mathbf{A}_{\gamma}^{+}=\text {Ind } \mathscr{A}_{-\gamma}-\operatorname{dim}\left(\mathbf{W}_{\gamma}^{1}(\Omega) \ominus\left(\mathbf{W}_{\gamma}^{1}(\Omega)\right)^{+}\right)=J-J=0 .
$$

Пусть в подпространстве ker $\mathscr{A}_{-\gamma}$ нет базиса, элементы которого допускают разложения (4.4). Тогда в нем найдется нетривиальный элемент $U \in\left(\mathbf{W}_{\gamma}^{1}(\Omega)\right)^{+}$. Подставим его в формулу Грина на области $\Omega(R)=\{x \in \Omega: z<R\}$ и получим

$$
0=\mathbf{q}(U, U)=\lim _{R \rightarrow+\infty} \mathbf{q}\left(\sum_{j=1}^{J} a_{j}^{+} w_{j}^{+}+\widetilde{U}, \sum_{j=1}^{J} a_{j}^{+} w_{j}^{+}+\widetilde{U}\right)=i \sum_{j=1}^{J}\left|a_{j}^{+}\right|^{2} .
$$

Экспоненциально малый остаток $\widetilde{U} \in W_{\gamma, 0}^{1}(\Omega)$ устранен предельным переходом, т. е. последнее равенство - следствие условий (4.3). Итак, все коэффициенты $a_{j}^{+}$аннулируются, а значит, $U \in \operatorname{ker} \mathscr{A}_{\gamma}=\{0\}$ и $U=0$. Аналогичные выкладки для функций (4.4) показывают, что форма $\mathbf{q}\left(Z_{j}, Z_{k}\right)$, равная нулю, принимает 
ВИД

$$
\lim _{R \rightarrow+\infty} \mathbf{q}\left(w_{j}^{-}+\sum_{m=1}^{J} S_{m j} w_{m}^{+}, w_{k}^{-}+\sum_{l=1}^{J} S_{l k} w_{l}^{+}\right)=-i \delta_{j, k}+i \sum_{m, l=1}^{J} S_{m j} \overline{S_{l k}} \delta_{m, l} .
$$

Таким образом, $S^{*} S=\mathbb{I}_{J}$ - единичная $(J \times J)$-матрица.

При $\varepsilon \in\left(-\varepsilon_{0}, \varepsilon_{0}\right)$ и малом $\varepsilon_{0}>0$ возмущенный оператор $\mathbf{A}_{\gamma}^{\varepsilon+}:\left(\mathbf{W}_{\gamma}^{1}(\Omega)\right)^{+} \rightarrow$ $\left(W_{-\gamma, 0}^{1}(\Omega)\right)^{*}$ остается изоморфизмом и, в свою очередь, порождает расширенную матрицу рассеяния $S^{\varepsilon}$, составленную из коэффициентов в следующих, аналогичных (4.4), формулах для решений $Z_{1}^{\varepsilon}, \ldots, Z_{J}^{\varepsilon}$ задачи (1.2):

$$
\mathbf{Z}_{j}^{\varepsilon}:=Z_{j}^{\varepsilon}-\chi w_{j}^{-}=\chi \sum_{k=1}^{J} S_{k j}^{\varepsilon} w_{k}^{+}+\widetilde{Z}_{j}^{\varepsilon}, \quad \widetilde{Z}_{j}^{\varepsilon} \in W_{\gamma, 0}^{1}(\Omega) .
$$

Подчеркнем, что благодаря компактности коэффициентов операторов (1.3) в формулах (4.4) и (4.5) фигурирует один и тот же набор волн.

Лемма 4. Матрица $S^{\varepsilon}$ аналитически зависит от малых параметров $\varepsilon$ и $\varepsilon \boldsymbol{\tau}^{\prime}$, где $\boldsymbol{\tau}^{\prime}=\left(\tau_{1}, \ldots, \tau_{N}\right)$. Кроме того, $S^{0}=S$.

Доказательство. Прежде всего, справедливо представление $\mathbf{A}_{\gamma}^{\varepsilon+}=\mathbf{A}_{\gamma}^{+}+$ $\mathbf{B}_{\gamma}^{+}(\varepsilon \boldsymbol{\tau})$, где $\mathbf{B}_{\gamma}^{+}(\varepsilon \boldsymbol{\tau}):\left(\mathbf{W}_{\gamma}^{1}(\Omega)\right)^{+} \rightarrow\left(W_{-\gamma, 0}^{1}(\Omega)\right)^{*}$ - непрерывное отображение, линейное относительно $\varepsilon \boldsymbol{\tau}$, причем $\left(\mathbf{B}_{\gamma}^{+}(\varepsilon \boldsymbol{\tau}) u, v\right)_{\Omega}=\varepsilon l(u, v)$. Далее, оператор $\mathbf{A}_{\gamma}^{+}$, а при малом $\varepsilon$ и оператор $\mathbf{A}_{\gamma}^{\varepsilon+}-$ изоморфизмы. Кроме того, разности (4.5) суть решения задач Дирихле в $\Omega$ для уравнений $-\Delta_{x} \mathbf{Z}_{j}^{\varepsilon}+\varepsilon L \mathbf{Z}_{j}^{\varepsilon}-\lambda \mathbf{Z}_{j}^{\varepsilon}=$ $\mathbf{F}_{j}:=[\Delta, \chi] w_{j}^{-} \in C_{c}^{\infty}(\bar{\Omega})$ (напоминаем, что $l\left(\chi w_{j}^{-}, v\right)=0$, согласно выбору срезающей функции $\chi$, коммутатор которой с лапласианом обозначен через $[\Delta, \chi])$. Наконец, коэффициенты разложений названных решений $\mathbf{Z}_{j}^{\varepsilon}=\left(\mathbf{A}_{\gamma}^{\varepsilon+}\right)^{-1} \mathbf{F}_{j} \in$ $\left(\mathbf{W}_{\gamma}^{1}(\Omega)\right)^{+}$вычисляются по формулам $S_{k j}^{\varepsilon}=-i \mathbf{q}\left(Z_{j}^{\varepsilon}-\chi w_{j}^{-}, \chi w_{k}^{+}\right)$, проистекающим из соотношений (4.3) и обеспечивающим нужную аналитичность.

Следующий критерий существования экспоненциально затухающих решений задачи (1.2) установлен в [6], но его краткое доказательство приводится для удобства читателя. Укажем нужные обозначения. Разобьем матрицу $S^{\varepsilon}$ на блоки:

$$
S^{\varepsilon}=\left(\begin{array}{cc}
S_{b b}^{\varepsilon} & S_{b \dagger}^{\varepsilon} \\
S_{\dagger b}^{\varepsilon} & S_{\dagger \dagger}^{\varepsilon}
\end{array}\right)
$$

Здесь $S_{b b}^{\varepsilon}$ и $S_{\dagger \dagger}^{\varepsilon}$ - матрицы с размерами $N \times N$ и $(J-N) \times(J-N)$ соответственно. Определим еще строки решений $Z_{b}^{\varepsilon}=\left(Z_{1}^{\varepsilon}, \ldots, Z_{N}^{\varepsilon}\right), Z_{\dagger}^{\varepsilon}=\left(Z_{N+1}^{\varepsilon}, \ldots, Z_{J}^{\varepsilon}\right)$ и $Z^{\varepsilon}=$ $\left(Z_{b}^{\varepsilon}, Z_{\dagger}^{\varepsilon}\right)$ и составим строки такого же строения из волн $w_{j}^{ \pm}$.

Теорема 5. (1) Справедливы равенства

$$
\operatorname{dim} \operatorname{ker}\left(S_{\dagger \dagger}^{\varepsilon}+\mathbb{I}_{J-N}\right)=\operatorname{dim} \operatorname{ker}\left(S^{\varepsilon}+\mathbb{I}_{J}\right)-\operatorname{dim} \operatorname{ker}\left(s^{\varepsilon}+\mathbb{I}_{N}\right)=\operatorname{dim} \operatorname{ker}\left(A^{\varepsilon}-\lambda\right) .
$$

Правая часть - кратность собственного числа $\lambda$, но нуль в случае $\lambda \notin \sigma_{p}^{\varepsilon}$.

(2) Унитарная матрица $S^{\varepsilon}$ симметрична, т.е. $S^{\varepsilon}=\left(S^{\varepsilon}\right)^{\top}$.

Доказательство. Новая запись формул (4.4) для решений $Z_{j}^{\varepsilon} \in W_{-\gamma, 0}^{1}(\Omega)$ возмущенной задачи (1.2) выглядит следующим образом:

$$
\left(Z_{b}^{\varepsilon}, Z_{\dagger}^{\varepsilon}\right)=\chi w^{-}+\chi w^{+} S^{\varepsilon}+\cdots=\chi\left(w_{b}^{-}, w_{\dagger}^{-}\right)+\chi\left(w_{b}^{+}, w_{\dagger}^{+}\right) S^{\varepsilon}+\cdots ;
$$


при этом многоточие заменяет элементы пространства $W_{\gamma, 0}^{1}(\Omega)$. Теперь второе утверждение обеспечено двумя наблюдениями: $\overline{w_{j}^{\ddagger}}=w_{j}^{\mp}$, согласно $(2.2),(4.2)$, и

$$
\overline{Z^{\varepsilon}}\left(\overline{S^{\varepsilon}}\right)^{-1}=\chi\left(\overline{w^{-}}+\overline{w^{+}} \overline{S^{\varepsilon}}\right)\left(\overline{S^{\varepsilon}}\right)^{-1}+\cdots=\chi\left(w^{+}\left(\overline{S^{\varepsilon}}\right)^{-1}+w^{-}\right)+\cdots
$$

Напоминаем, что коэффициенты всех дифференциальных операторов вещественные. Строка (4.9) решений задачи (1.2) обладает таким же набором приходящих волн, что и у строки (4.8). По теореме 3 эти строки совпадают, и выполнено соотношение $\overline{S^{\varepsilon}}=\left(S^{\varepsilon}\right)^{-1}=\left(\overline{S^{\varepsilon}}\right)^{\top}$, влекущее за собой второе утверждение.

Пусть $a_{\dagger}^{\varepsilon} \in \operatorname{ker}\left(S_{\dagger \dagger}^{\varepsilon}+\mathbb{I}_{J-N}\right)$. Дополним столбец сверху нулями до высоты $J-$ обозначения $a_{b}^{\varepsilon}=0 \in \mathbb{C}^{N}$ и $a^{\varepsilon} \in \mathbb{C}^{J}$ понятны. В силу унитарности симметричной матрицы (4.6) и равенства $S_{\dagger \dagger}^{\varepsilon} a_{\dagger}^{\varepsilon}+a_{\dagger}^{\varepsilon}=0$ справедливы соотношения

$$
\begin{gathered}
\left|a_{\dagger}^{\varepsilon}\right|^{2}=\left|a^{\varepsilon}\right|^{2}=\left|S^{\varepsilon} a^{\varepsilon}\right|^{2}=\left|S_{b \dagger}^{\varepsilon} a_{\dagger}^{\varepsilon}\right|^{2}+\left|S_{\dagger \dagger}^{\varepsilon} a_{\dagger}^{\varepsilon}\right|^{2}=\left|S_{b \dagger}^{\varepsilon} a_{\dagger}^{\varepsilon}\right|^{2}+\left|a_{\dagger}^{\varepsilon}\right|^{2}, \\
S_{b \dagger}^{\varepsilon} a_{\dagger}^{\varepsilon}=0 \in \mathbb{C}^{N} \quad \text { при } a_{\dagger}^{\varepsilon} \in \operatorname{ker}\left(S_{\dagger \dagger}^{\varepsilon}+\mathbb{I}_{J-N}\right) .
\end{gathered}
$$

Формула (4.10) имеет два важных последствия. Во-первых,

$$
\begin{aligned}
Z^{\varepsilon} a^{\varepsilon} & =Z_{\dagger}^{\varepsilon} a_{\dagger}^{\varepsilon}=\chi\left(w_{\dagger}^{-} a_{\dagger}^{\varepsilon}+w_{b}^{+} S_{b \dagger}^{\varepsilon} a_{\dagger}^{\varepsilon}+w_{\dagger}^{+} S_{\dagger \dagger}^{\varepsilon} a_{\dagger}^{\varepsilon}\right)+\cdots=\chi\left(w_{\dagger}^{-} a_{\dagger}^{\varepsilon}-w_{\dagger}^{+} a_{\dagger}^{\varepsilon}\right)+\cdots \\
& =2^{-1 / 2} \chi\left(\left(v_{\dagger}^{+}+i v_{\dagger}^{-}\right) a_{\dagger}^{\varepsilon}-\left(v_{\dagger}^{+}-i v_{\dagger}^{-}\right) a_{\dagger}^{\varepsilon}\right)+\cdots=2^{1 / 2} i \chi v_{\dagger}^{-} a_{\dagger}^{\varepsilon}+\cdots
\end{aligned}
$$

т. е. $Z^{\varepsilon} a^{\varepsilon} \in W_{\beta, 0}^{1}(\Omega)$ - захваченная волна, так как функции $(2.3)$ в строке $v_{\dagger}^{-}$ экспоненциально затухают. Во-вторых, соотношение (4.10) вместе с равенством $S_{\dagger b}^{\varepsilon}=\left(S_{b \dagger}^{\varepsilon}\right)^{\top}$ показывает, что $(N \times N)$-матрица $\Sigma^{\varepsilon}=-S_{b \dagger}^{\varepsilon}\left(S_{\dagger \dagger}^{\varepsilon}+\mathbb{I}_{J-N}\right)^{-1} S_{\dagger b}^{\varepsilon}$ определена корректно: под $\left(S_{\dagger \dagger}^{\varepsilon}+\mathbb{I}_{J-N}\right)^{-1}$ понимаем обратный оператор, определенный на образе $\left(S_{\dagger \dagger}^{\varepsilon}+\mathbb{I}_{J-N}\right) \mathbb{C}^{J-N}$ и действующий на ортогональное дополнение $\operatorname{ker}\left(S_{\dagger \dagger}^{\varepsilon}+\mathbb{I}_{J-N}\right)$ до $\mathbb{C}^{J-N}$. Если $a_{b}^{\varepsilon} \in \mathbb{C}^{N}-$ собственный вектор матрицы $s^{\varepsilon}$, отвечающий ее собственному числу -1 , то столбец $a^{\varepsilon}$, составленный из $a_{b}^{\varepsilon} \in \mathbb{C}^{N}$ и $a_{\dagger}^{\varepsilon}=-\left(S_{\dagger \dagger}^{\varepsilon}+\mathbb{I}_{J-N}\right)^{-1} S_{\dagger b}^{\varepsilon} a_{b}^{\varepsilon} \in \mathbb{C}^{J-N}$, - собственный для матрицы $S^{\varepsilon}$, отвечающий тому же собственному числу -1 и ортогональный подпространству $\{0\}^{N} \times \operatorname{ker}\left(S_{\dagger \dagger}^{\varepsilon}+\mathbb{I}_{J-N}\right)$ по определению обратного оператора $\left(S_{\dagger \dagger}^{\varepsilon}+\mathbb{I}_{J-N}\right)^{-1}$. Эти факты обеспечивают первое равенство в (4.7).

Связь $s^{\varepsilon}=S_{b b}^{\varepsilon}+\Sigma^{\varepsilon}$ матриц рассеяния, обычной и расширенной, устанавливается сравнением разложений (3.3) и

$$
\begin{aligned}
Z_{b}^{\varepsilon}-Z_{\dagger}^{\varepsilon}\left(S_{\dagger \dagger}^{\varepsilon}+\right. & \left.\mathbb{I}_{J-N}\right)^{-1} S_{\dagger b}^{\varepsilon}=\chi w_{b}^{-}+\chi w_{b}^{+}\left(S_{b b}^{\varepsilon}-S_{b \dagger}^{\varepsilon}\left(S_{\dagger \dagger}^{\varepsilon}+\mathbb{I}_{J-N}\right)^{-1} S_{\dagger b}^{\varepsilon}\right) \\
& +2^{-1 / 2} \chi v_{\dagger}^{+}\left(S_{\dagger b}^{\varepsilon}-\left(\mathbb{I}_{J-N}+S_{\dagger \dagger}^{\varepsilon}\right)\left(S_{\dagger \dagger}^{\varepsilon}+\mathbb{I}_{J-N}\right)^{-1} S_{\dagger b}^{\varepsilon}\right)+\cdots
\end{aligned}
$$

В формуле (4.11) (а также и в следующей) многоточие заменяет слагаемое из весового класса $W_{\beta, 0}^{1}(\Omega)$. Правый множитель при $v_{\dagger}^{+}$обращается в нуль и возникает при извлечении растущих волн $\chi v_{\dagger}^{+}$из линейных комбинаций $(4.2)$, а множитель при $w_{b}^{+}$совпадает с матрицей рассеяния $s^{\varepsilon}$, так как строка (4.11) может отличаться от строки $\zeta^{\varepsilon}$ лишь слагаемыми из подпространства ker $\mathscr{A}_{\beta}$, не влияющими на коэффициенты при осциллирующих волнах $w_{b}^{ \pm}$. 
Наконец, если решение $Z^{\varepsilon} a^{\varepsilon}$ задачи (1.2) исчезает при $z \rightarrow+\infty$, то в формуле

$$
\begin{aligned}
Z^{\varepsilon} a^{\varepsilon} & =Z_{b}^{\varepsilon} a_{b}^{\varepsilon}+Z_{\dagger}^{\varepsilon} a_{\dagger}^{\varepsilon} \\
& =\chi w_{b}^{-} a_{b}^{\varepsilon}+\chi w_{b}^{+}\left(S_{b b}^{\varepsilon} a_{b}^{\varepsilon}+S_{b \dagger}^{\varepsilon} a_{\dagger}^{\varepsilon}\right)+2^{-1 / 2} \chi v_{\dagger}^{+}\left(a_{\dagger}^{\varepsilon}+S_{\dagger \dagger}^{\varepsilon} a_{\dagger}^{\varepsilon}+S_{\dagger b}^{\varepsilon} a_{b}^{\varepsilon}\right)+\cdots
\end{aligned}
$$

обращаются в нуль множители при осциллирующих волнах $w_{b}^{-}, w_{b}^{+}$и экспоненциально растущих волнах $v_{+}^{+}$. Первое и третье требования приводят к соотношениям $a_{b}^{\varepsilon}=0$ и $S_{\dagger \dagger}^{\varepsilon} a_{\dagger}^{\varepsilon}+a_{\dagger}^{\varepsilon}=0$, но тогда второе выполнено благодаря (4.10). Итак, проверены оба равенства в (4.7).

Замечание 6. Формула (4.7) верна лишь при условии $\operatorname{dim} \mathrm{ker} \mathscr{A}_{\gamma}^{\varepsilon}=0$. Если же оно нарушено, то положительность правой части формулы (4.7) перестает быть критерием существования захваченных волн, так как матрица $S^{\varepsilon}$ не содержит информации о «сверхбыстро» затухающих при $z \rightarrow+\infty$ решениях. Игнорирование этого обстоятельства в вычислительных схемах приводит к ошибкам (они обсуждаются, например, в статье [13]), и в общей ситуации правильно применять безусловный критерий [13], оперирующий с оператором фиктивного рассеяния - бесконечномерным аналогом расширенной матрицы рассеяния.

\section{§5. Подготовительная работа}

Пусть собственное число (1.11) задачи (1.4) простое. Зафиксируем числа $J$ и $\gamma$ из (2.5) так, чтобы соответствующая собственная функция $\widehat{u}^{0} \in H_{0}^{1}(\Omega)$, вещественная и нормированная в $L_{2}(\Omega)$, имела вид

$$
\widehat{u}^{0}=\chi \widehat{a}^{0} v_{J}^{-}+\widetilde{u}^{0} \quad\left(\widehat{a}^{0} \neq 0, \widetilde{u}^{0} \in W_{\gamma, 0}^{1}(\Omega)\right),
$$

предположив дополнительно для простоты (ср. конец $§ 7)$, что собственное число $\Lambda_{J}$ задачи $(1.8)$ также простое. Соорудим новое пространство $\left(\widehat{\mathbf{W}}_{\gamma}^{01}(\Omega)\right)^{+}$с отделенной асимптотикой из функций, допускающих представление

$$
u=\chi\left(\sum_{n=1}^{N} a_{n}^{+} w_{n}^{+}+a_{J} w_{J}^{+}\right)+\widetilde{u} \quad\left(\widetilde{u}=\chi \sum_{j=N+1}^{J-1} b_{n}^{-} v_{n}^{-}+\widetilde{\widetilde{u}}, \widetilde{\widetilde{u}} \in W_{\gamma, 0}^{1}(\Omega)\right) .
$$

Оба остатка $\widetilde{u} \in W_{\beta, 0}^{1}(\Omega)$ и $\widetilde{\widetilde{u}} \in W_{\gamma, 0}^{1}(\Omega)$ экспоненциально затухают, однако по сравнению с элементами пространства (3.2) разложение функции $u$ из формулы (5.2) включает волновой пакет $w_{J}^{+}$, порожденный главным членом асимптотики (5.1) собственной функции $\widehat{u}^{0}$. То же самое соотношение для $u$ можно получить заменой в разложении функции из класса $\left(\mathbf{W}_{\gamma}^{1}(\Omega)\right)^{+}$волновых пакетов $w_{N+1}^{-}, \ldots, w_{J-1}^{-}$их экспоненциально затухающими составляющими $v_{N+1}^{-}, \ldots, v_{J-1}^{-}($ср. определение $(4.2))$, которые отсутствуют в асимптотике $(5.1)$ собственной функции $\widehat{u}^{0}$ - именно поэтому при анализе устойчивости простого собственного числа (1.11) без них можно обойтись. Вместе с тем, как и ранее $\mathbf{A}_{\gamma}^{+}$ в $\$ 4$, сужение $\widehat{\mathbf{A}}_{\gamma}^{0+}$ оператора $\mathscr{A}_{-\gamma}$ с $W_{-\gamma, 0}^{1}(\Omega)$ на $\left(\widehat{\mathbf{W}}_{\gamma}^{01}(\Omega)\right)^{+}$оказывается изоморфизмом. Дело в том, что по предположениям о параметре $\lambda$ и собственной функции (5.1) оператор $\widehat{\mathbf{A}}_{\gamma}^{0+}$ - мономорфизм, а затухающие волны аннулируют форму (4.1), и поэтому новое качество остатка $\widetilde{u}$ в разложении (5.2) не препятствует буквальному повторению доказательства обоих пунктов теоремы 3. 
Введем обозначения $\widehat{w}_{j}^{0 \pm}$ для волн (4.2). Видоизмененные разложения (4.4) новых решений $\widehat{Z}_{j}^{0}$ задачи (1.2) принимают вид

$$
\widehat{Z}_{j}^{\varepsilon}=\chi \widehat{w}_{j}^{\varepsilon-}+\chi\left(\sum_{n=1}^{N} \widehat{S}_{n j}^{\varepsilon} \widehat{w}_{n}^{\varepsilon+}+\widehat{S}_{J j}^{\varepsilon} \widehat{w}_{J}^{\varepsilon+}\right)+\widetilde{Z}_{j}^{\varepsilon}, \quad j=1, \ldots, N, J,
$$

где пока следует положить $\varepsilon=0$. При этом $\widehat{\mathbf{Z}}_{j}^{0}=\widehat{Z}_{j}^{0}-\chi \widehat{w}_{j}^{0-} \in\left(\widehat{\mathbf{W}}_{\gamma}^{01}(\Omega)\right)^{+}$, остатки $\widetilde{Z}_{j}^{0}$ приобретают те же качества, что и ранее $\widetilde{u}$ в $(5.2)$, а коэффициенты $\widehat{S}_{k j}^{0}$ образуют унитарную матрицу $\widehat{S}^{0}$ размера $(N+1) \times(N+1)$. Упомянутые решения определены равенствами

$$
\widehat{Z}_{n}^{0}=\zeta_{n}, \quad n=1, \ldots, N, \quad \widehat{Z}_{J}^{0}=2^{1 / 2} i\left(\widehat{a}^{0}\right)^{-1} \widehat{u}^{0},
$$

где $\zeta_{n}$ - решение, указанное предложением 2 , причем благодаря имеющемуся произволу в выборе $\zeta_{n}, n=1, \ldots, N,-$ слагаемому $c_{n} \widehat{u}^{0}-$ коэффициенты при волне $v_{J}^{-}$сделаны нулевыми. В итоге, согласно соотношениям (3.3) и (5.1), имеем

$$
\widehat{S}_{n m}^{0}=s_{n m}, \quad \widehat{S}_{n J}^{0}=\widehat{S}_{J n}^{0}=0, \quad n, m=1, \ldots, N, \quad \widehat{S}_{J J}^{0}=-1 .
$$

Сокращение размеров новой расширенной матрицы рассеяния $\widehat{S}^{0}$ и явные формулы (5.5) значительно упрощают выкладки. Удобно использовать такое разбиение матрицы (как и в определении (5.3) считаем, что пока в нем $\varepsilon=0$ ):

$$
\widehat{S}^{\varepsilon}=\left(\begin{array}{cc}
\widehat{S}_{b b}^{\varepsilon} & \widehat{S}_{b J}^{\varepsilon} \\
\widehat{S}_{J b}^{\varepsilon} & \widehat{S}_{J J}^{\varepsilon}
\end{array}\right) .
$$

По спектральному параметру

$$
\lambda^{\varepsilon}=\lambda+\varepsilon \mu \quad\left(\mu \in\left[-\mu_{0}, \mu_{0}\right]\right)
$$

определим аналогичные $(2.2),(2.3)$ и (4.2) волны $\widehat{w}_{1}^{\varepsilon \pm}, \ldots, \widehat{w}_{N}^{\varepsilon \pm}, \widehat{v}_{N+1}^{\varepsilon \pm}, \ldots, \widehat{v}_{J-1}^{\varepsilon \pm}$ и $\widehat{w}_{J}^{\varepsilon \pm}$. Если параметр $\varepsilon$ мал, а величина $\mu_{0}>0$ и компоненты вектора $\boldsymbol{\tau}^{\prime}$ равномерно ограничены, то оператор $\widehat{\mathbf{A}}_{\gamma}^{\varepsilon+}$, являясь малым возмущением оператора $\widehat{\mathbf{A}}_{\gamma}^{0+}$, остается изоморфизмом, причем его норма и норма обратного к нему оператора равномерно ограничены относительно параметров $\Theta=\left(\tau_{1}, \ldots, \tau_{N}, \mu\right) \in$ $\mathbb{B}_{\rho}^{N+1}$ (шар с некоторым фиксированным радиусом $\rho>0$ ). Проверка очередного утверждения дословно повторяет доказательства леммы 4 и теоремы 5.

Лемма 7. Новая расширенная матрица рассеяния $\widehat{S}^{\varepsilon}$ из (5.6) аналитически зависит от малых $\varepsilon, \varepsilon \mu$ и $\varepsilon \boldsymbol{\tau}^{\prime}=\left(\varepsilon \tau_{1}, \ldots, \varepsilon \tau_{N}\right)$. Кроме того, $\widehat{S}^{\varepsilon}=\left(\widehat{S}^{\varepsilon}\right)^{\top}$.

Найдем асимптотику столбца $\widehat{S}_{J}^{\varepsilon}$ матрицы (5.6), порожденного решением $\widehat{Z}_{J}^{\varepsilon}$ задачи (1.5) и состоящего из столбца $\widehat{S}_{b J}^{\varepsilon} \in \mathbb{C}^{N}$ и скаляра $\widehat{S}_{J J}^{\varepsilon} \in \mathbb{C}$. Оценки

$$
\left|\widehat{S}_{n m}^{\varepsilon}-s_{n m}\right| \leqslant c \varepsilon
$$

элементов симметричной подматрицы $\widehat{S}_{\mathrm{bb}}^{\varepsilon}$ обеспечены леммой 7 и равенствами (5.5); при этом множитель $c$ не зависит от $\Theta \in \mathbb{B}_{\rho}^{N+1}$. В силу соотношений (5.7) и $(1.3),(1.6)$ сами решения (5.3) и поправочные слагаемые $S_{j J}^{\prime}$ в формуле

$$
\widehat{S}^{\varepsilon}=\widehat{S}^{0}+\varepsilon S^{\prime}+\widetilde{S}^{\varepsilon}, \quad\left|\widetilde{S}^{\varepsilon}\right| \leqslant c \varepsilon^{2},
$$


принимают соответственно вид

$$
\begin{gathered}
\widehat{Z}_{J}^{\varepsilon}=\widehat{Z}_{J}^{0}+\varepsilon Z_{J}^{\prime}+\widetilde{Z}_{J}^{\varepsilon}, \quad Z_{J}^{\prime}=Z_{J}^{(0)}+\tau_{1} Z_{J}^{(1)}+\cdots+\tau_{N} Z_{J}^{(N)}+\mu Z_{J}^{(\mu)}, \\
S_{j J}^{\prime}=S_{j J}^{(0)}+\tau_{1} S_{j J}^{(1)}+\cdots+\tau_{N} S_{j J}^{(N)}+\mu S_{j J}^{(\mu)}, \quad j=1, \ldots, N, J
\end{gathered}
$$

при этом $\widetilde{Z}_{J}^{\varepsilon}$ - малый остаток, который еще предстоит оценить. Кроме того, $S_{j J}^{(n)}, n=0, \ldots, N,-$ множители при $\widehat{w}_{j}^{0+}$ в решении $Z_{J}^{(n)} \in\left(\widehat{\mathbf{W}}_{\gamma}^{01}(\Omega)\right)^{+}$задачи

$$
\left(\nabla Z_{J}^{(n)}, \nabla v\right)_{\Omega}-\lambda\left(Z_{J}^{(n)}, v\right)_{\Omega}=-l_{n}\left(\widehat{Z}_{J}^{0}, v\right), \quad v \in W_{-\gamma, 0}^{1}(\Omega) .
$$

Наконец, последняя функция в $(5.10)$ и коэффициенты $\mu S_{j J}^{(\mu)}$ ее разложения в (5.11) появляются из-за возмущения (5.7) спектрального параметра. Подставив (5.10) и (5.7) в равенства (1.2) и выделив множители при $\mu$, приходим к задаче

$$
-\Delta_{x} Z_{J}^{(\mu)}-\lambda Z_{J}^{(\mu)}=\widehat{Z}_{J}^{0}=\sqrt{2} i\left(\widehat{a}^{0}\right)^{-1} \widehat{u}^{0} \quad \text { в } \Omega, \quad Z_{J}^{(\mu)}=0 \quad \text { на } \partial \Omega .
$$

Изучим решения указанных задач. Поскольку носители коэффициентов дифференциальных операторов (1.3) компактны, решения $Z_{J}^{(n)} \in\left(\widehat{\mathbf{W}}_{\gamma}^{01}(\Omega)\right)^{+}$задач (5.12) удовлетворяют уравнению Гельмгольца в полуцилиндре $Q_{\ell}$ и обращаются в нуль на его боковой поверхности, а значит, для вычисления коэффициентов $S_{j J}^{(n)}$ при волнах $\widehat{w}_{j}^{0+}$ в их разложениях можно применить форму (4.1). Так, при помощи соотношений $(4.3),(5.4)$ и (3.3) получаем, что

$$
\begin{aligned}
& 2^{1 / 2} i\left(\widehat{a}^{0}\right)^{-1} l_{n}\left(\widehat{u}^{0}, \zeta_{k}\right)=l_{n}\left(\widehat{Z}_{J}^{0}, \zeta_{k}\right)=\mathbf{q}\left(Z_{J}^{(n)}, \zeta_{k}\right) \\
= & \mathbf{q}\left(\sum_{p=1}^{N} S_{p J}^{(n)} \widehat{w}_{p}^{0+}+S_{J J}^{(n)} \widehat{w}_{J}^{0+}+\widetilde{Z}_{J}^{(n)}, \widehat{w}_{k}^{0-}+\sum_{m=1}^{N} s_{m k} \widehat{w}_{m}^{0+}+\widetilde{\zeta}_{k}\right)=i \sum_{m=1}^{N} S_{m J}^{(n)} \overline{s_{m k}} .
\end{aligned}
$$

Отсюда и из аналогичной выкладки с заменой $\zeta_{k} \mapsto \widehat{u}^{0}=-2^{-1 / 2} i \widehat{a}^{0} \widehat{Z}_{J}^{0}$ выводим равенства

$$
\begin{gathered}
\sum_{m=1}^{N} S_{m J}^{(n)} \overline{s_{m k}}=2^{1 / 2}\left(\widehat{a}^{0}\right)^{-1} l_{n}\left(\widehat{u}^{0}, \zeta_{k}\right), \quad k=1, \ldots, N, \\
S_{J J}^{(n)}=2 i\left|\widehat{a}^{0}\right|^{-2} l_{n}\left(\widehat{u}^{0}, \widehat{u}^{0}\right), \quad n=0, \ldots, N .
\end{gathered}
$$

Член $\sqrt{2} i \chi v_{J}^{-}$, порожденный асимптотикой (5.1), делает правую часть формулы (5.13) убывающей недостаточно быстро. Однако этот главный асимптотический член компенсируется выражением $\mathscr{Z}=i\left(2\left(\Lambda_{J}-\lambda\right)\right)^{-1 / 2} z v_{J}^{-}$, решающим задачу

$$
-\Delta \mathscr{Z}-\lambda \mathscr{Z}=\sqrt{2} i v_{J}^{-} \quad \text { в } Q, \quad \mathscr{Z}=0 \quad \text { на } \partial Q,
$$

а оставшаяся правая часть попадает в пространство $\left(W_{-\gamma, 0}^{1}(\Omega)\right)^{*}$, т. е. затухает достаточно быстро. В итоге задача для разности $Z_{J}^{(\mu)}-\chi \mathscr{Z}$ однозначно разрешима в классе $\left(\widehat{\mathbf{W}}_{\gamma}^{01}(\Omega)\right)^{+}$, т. е. решение $Z_{J}^{(\mu)}$ задачи (5.13) действительно существует. Появление в его асимптотике слагаемого $\mathscr{Z}$ согласовано с формулой 
Тейлора

$$
e^{-z \sqrt{\Lambda_{J}-\lambda-\varepsilon \mu}} U_{j}(y)=e^{-z \sqrt{\Lambda_{J}-\lambda}}\left(1+\frac{\varepsilon \mu z}{2 \sqrt{\Lambda_{J}-\lambda}}+O\left(\varepsilon^{2}\right)\right) U_{j}(y), \quad|z| \leqslant R,
$$

для волны $\widehat{v}_{J}^{\varepsilon-}(x)$ (в определении (2.3) произведена замена $\lambda \mapsto \lambda+\varepsilon \mu$ ). Коэффициенты при осциллирующих волнах $\widehat{w}_{1}^{0+}, \ldots, \widehat{w}_{N}^{0+}$ и экспоненциальной волне $\widehat{w}_{J}^{0+}$ в разложении решения $Z_{J}^{(\mu)}$ вычисляются по прежней схеме, так как член $\mathscr{Z}$ экспоненциально мал. В результате приходим к похожим на (5.14) соотношениям, однако вместо формы (1.6) в них фигурирует скалярное произведение $(\cdot, \cdot)_{\Omega}$ :

$$
\begin{gathered}
\sum_{m=1}^{N} S_{m J}^{(\mu)} \overline{s m k}=-2^{1 / 2}\left(\widehat{a}^{0}\right)^{-1}\left(\widehat{u}^{0}, \zeta_{k}\right)_{\Omega}, \quad k=1, \ldots, N, \\
S_{J J}^{(\mu)}=-2 i\left|\widehat{a}^{0}\right|^{-2}\left(\widehat{u}^{0}, \widehat{u}^{0}\right)_{\Omega}, \quad n=0, \ldots, N .
\end{gathered}
$$

В итоге поправочное слагаемое из разложения (5.10) принимает вид

$$
Z_{J}^{\prime}=\chi\left(\widehat{w}_{J}^{0+} S_{J J}^{\prime}+\sum_{j=1}^{N} \widehat{w}_{j}^{0+} S_{j J}^{\prime}+\sum_{p=N+1}^{J-1} b_{p}^{\prime} \widehat{v}_{j}^{0-}+\mu \mathscr{Z}\right)+\widetilde{Z}_{J}^{\prime}, \quad \widetilde{Z}_{J}^{\prime} \in W_{\gamma, 0}^{1}(\Omega) .
$$

Для оправдания расчетов построим глобальное асимптотическое приближение $\widehat{Z}_{\text {Jas }}^{\varepsilon}$ к решению $\widehat{Z}_{J}^{\varepsilon}$ задачи (1.2) с параметром (5.7) при помощи конструкции с «перехлестывающимися» срезками $\chi \in C^{\infty}(\mathbb{R})$ и $X_{\varepsilon}$, обычной для метода составных асимптотических разложений (см. [14, гл. 2]). Первая срезка была определена формулами (2.4), а вторая имеет вид $X_{\varepsilon}(z)=1-\chi\left(z-\varepsilon^{-1}\right)$. Напомним также, что коэффициенты дифференциального оператора (1.3) обращаются в нуль на носителе $\chi$. Положим

$$
\begin{gathered}
\widehat{Z}_{\mathrm{Jas}}^{\varepsilon}=\chi \widehat{Z}_{J \infty}^{\varepsilon}+X_{\varepsilon} \widehat{Z}_{J 0}^{\varepsilon}-\chi X_{\varepsilon} \widehat{Z}_{J 0 \infty}^{\varepsilon}, \\
\widehat{Z}_{J \infty}^{\varepsilon}=\widehat{w}_{J}^{\varepsilon-}-\widehat{w}_{J}^{\varepsilon+}+\varepsilon\left(\widehat{w}_{J}^{\varepsilon+} S_{J J}^{\prime}+\sum_{n=1}^{N} \widehat{w}_{n}^{\varepsilon+} S_{n J}^{\prime}+\sum_{p=N+1}^{J-1} b_{p}^{\prime} \widehat{v}_{p}^{\varepsilon-}\right), \quad \widehat{Z}_{J 0}^{\varepsilon}=\widehat{Z}_{J}^{0}+\varepsilon Z_{J}^{\prime}, \\
\widehat{Z}_{J 0 \infty}^{\varepsilon}=\widehat{w}_{J}^{0-}-\widehat{w}_{J}^{0+}+\varepsilon\left(\widehat{w}_{J}^{0+} S_{J J}^{\prime}+\sum_{n=1}^{N} \widehat{w}_{n}^{0+} S_{n J}^{\prime}+\sum_{p=N+1}^{J-1} b_{p}^{\prime} \widehat{v}_{p}^{0-}+\mu \mathscr{Z}\right),
\end{gathered}
$$

а коэффициенты $S_{q J}^{\prime}$ и $b_{p}^{\prime}$ возьмем из разложения (5.18). Обращаем внимание на то, что выражение $\widehat{Z}_{J 0 \infty}^{\varepsilon}$ является фрагментом асимптотики обоих слагаемых $\widehat{Z}_{J \infty}^{\varepsilon}$ и $\widehat{Z}_{J 0}^{\varepsilon}$ в правой части формулы (5.19), однако выражение $-\chi X_{\varepsilon} \widehat{Z}_{J 0 \infty}^{\varepsilon}$ устраняет дублирование. Кроме того, при коммутировании оператора Лапласа со срезками присоединяем это выражение либо к $\chi \widehat{Z}_{J \infty}^{\varepsilon}$, либо к $X_{\varepsilon} \widehat{Z}_{J 0}^{\varepsilon}$, уменьшая тем самым невязку. В результате оказывается, что при любой функции 
$v \in C_{c}^{\infty}(\Omega)$ разность $\widehat{Z}_{J}^{\varepsilon}-\widehat{Z}_{\mathrm{Jas}}^{\varepsilon}$ удовлетворяет тождеству

$$
\begin{aligned}
\left(\nabla\left(\widehat{Z}_{J}^{\varepsilon}-\widehat{Z}_{\mathrm{Jas}}^{\varepsilon}\right), \nabla v\right)_{\Omega}+\varepsilon l\left(\widehat{Z}_{J}^{\varepsilon}-\widehat{Z}_{\mathrm{Jas}}^{\varepsilon}, v\right)-(\lambda+\varepsilon \mu)\left(\widehat{Z}_{J}^{\varepsilon}-\widehat{Z}_{\mathrm{Jas}}^{\varepsilon}, v\right)_{\Omega} \\
=I^{\infty}+I^{0}+I^{0 \infty}+I_{\mathrm{com}}^{\infty}+I_{\mathrm{com}}^{0} \\
:=-\left(\nabla \widehat{Z}_{J \infty}^{\varepsilon}, \nabla(\chi v)\right)_{\Omega}+(\lambda+\varepsilon \mu)\left(\widehat{Z}_{J \infty}^{\varepsilon}, \chi v\right)_{\Omega} \\
\quad-\left(\nabla \widehat{Z}_{J 0}^{\varepsilon}, \nabla\left(X_{\varepsilon} v\right)\right)_{\Omega}-\varepsilon l\left(\widehat{Z}_{J 0}^{\varepsilon}, X_{\varepsilon} v\right)+(\lambda+\varepsilon \mu)\left(\widehat{Z}_{J 0}^{\varepsilon}, X_{\varepsilon} v\right)_{\Omega} \\
\quad+\left(\nabla \widehat{Z}_{J 0 \infty}^{\varepsilon}, \nabla\left(\chi X_{\varepsilon} v\right)\right)_{\Omega}-(\lambda+\varepsilon \mu)\left(\widehat{Z}_{J 0 \infty}^{\varepsilon}, \chi X_{\varepsilon} v\right)_{\Omega} \\
\quad-\left(\left(\widehat{Z}_{J \infty}^{\varepsilon}-\widehat{Z}_{J 0 \infty}^{\varepsilon}\right) \nabla \chi, \nabla v\right)_{\Omega}+\left(\nabla\left(\widehat{Z}_{J \infty}^{\varepsilon}-\widehat{Z}_{J 0 \infty}^{\varepsilon}\right), v \nabla \chi\right)_{\Omega} \\
\quad-\left(\left(\widehat{Z}_{J 0}^{\varepsilon}-\widehat{Z}_{J 0 \infty}^{\varepsilon}\right) \nabla X_{\varepsilon}, \nabla v\right)_{\Omega}+\left(\nabla\left(\widehat{Z}_{J 0}^{\varepsilon}-\widehat{Z}_{J 0 \infty}^{\varepsilon}\right), v \nabla X_{\varepsilon}\right)_{\Omega} .
\end{aligned}
$$

Подробные объяснения несложных и рутинных преобразований, приведших к формуле (5.20) и обеспечивающих дальнейшие выводы, приведены, например, в $[14$, гл. 2]. Укажем лишь, что, согласно сделанным предположениям, $l(Z, \chi v)=0$ и $l\left(Z, X_{\varepsilon} v\right)=l(Z, v)$ при любых приемлемых функциях $Z$ и $v$.

Сразу заметим, что $I^{\infty}=0$ по определению волн $\widehat{w}_{q}^{\varepsilon \pm}$ и $\widehat{v}_{p}^{\varepsilon-}$. Волны $\widehat{w}_{q}^{0 \pm}$ и $\widehat{v}_{p}^{0-}$ аннулируются оператором $\Delta+\lambda$; поэтому, согласно $(5.15)$, имеем

$$
\begin{gathered}
\left(\nabla\left(\widehat{Z}_{J 0 \infty}^{\varepsilon}-\varepsilon \mu \mathscr{Z}\right), \nabla\left(\chi X_{\varepsilon} v\right)\right)_{\Omega}-\lambda\left(\widehat{Z}_{J 0 \infty}^{\varepsilon}-\varepsilon \mu \mathscr{Z}, \chi X_{\varepsilon} v\right)_{\Omega}=0 \\
\left(\nabla \mathscr{Z}, \nabla\left(\chi X_{\varepsilon} v\right)\right)_{\Omega}-\lambda\left(\mathscr{Z}, \chi X_{\varepsilon} v\right)_{\Omega}=\left(\widehat{w}_{J}^{0-}-\widehat{w}_{J}^{0+}, \chi X_{\varepsilon} v\right)_{\Omega} .
\end{gathered}
$$

Оставшуюся в выражении $I^{0 \infty}$ часть

$$
I_{1}^{0 \infty}=-\varepsilon^{2} \mu\left(\chi\left(\widehat{w}_{J}^{0+} S_{J J}^{\prime}+\sum_{n=1}^{N} \widehat{w}_{n}^{0+} S_{n J}^{\prime}+\sum_{p=N+1}^{J-1} b_{p}^{\prime} \widehat{v}_{p}^{0-}+\mu \mathscr{Z}\right), X_{\varepsilon} v\right)_{\Omega}
$$

присоединим к $I^{0}$. Учитывая формулы $(5.12),(5.13),(5.18)$ и $(5.10)$ для $Z_{J}^{\prime}$, находим:

$$
I^{0}+I_{1}^{0 \infty}=-\varepsilon^{2} l\left(Z_{J}^{\prime}, v\right)+\varepsilon^{2} \mu\left(\widetilde{Z}_{J}^{\prime}, X_{\varepsilon} v\right)_{\Omega}, \quad\left|I^{0}+I_{1}^{0 \infty}\right| \leqslant c \varepsilon^{2}\left\|v ; W_{-\gamma, 0}^{1}(\Omega)\right\| .
$$

Оценка с весовой нормой $\left\|v ; W_{-\gamma, 0}^{1}(\Omega)\right\|$ стала возможной благодаря приведенному в (5.18) включению для $\widetilde{Z}_{J}^{\prime}$ и упомянутым выше свойствам форм (1.6).

Производные срезок $\chi$ и $X_{\varepsilon}$ отличаются от нуля только в зонах $z \in[\ell, \ell+1]$ и $z \in\left[\ell+\varepsilon^{-1}, \ell+1+\varepsilon^{-1}\right]$ соответственно. Внутри первой зоны верна формула Тейлора (5.16), обеспечивающая малость невязки. Внутри второй учитываем экспоненциальное затухание разности $\widehat{Z}_{J 0}^{\varepsilon}-\widehat{Z}_{J 0 \infty}^{\varepsilon}=2^{1 / 2} i\left(\widehat{a}^{0}\right)^{-1} \widetilde{u}^{0}+\varepsilon \widetilde{Z}_{J}^{\prime} \in W_{\gamma, 0}^{1}(\Omega)$, более того, пользуемся возможностью увеличить весовой показатель $\gamma$. Дело в том, что функции $\widehat{u}^{0}$ и $Z_{J}^{(n)}$ являются решениями задачи $(2.1)$ с нулевой и финитными правыми частями, которые попадают в $\left(W_{-\gamma^{\prime}, 0}^{1}(\Omega)\right)^{*}$ при любом $\gamma^{\prime} \in \Upsilon(\lambda)$ (см. ограничение $\left.(2.5)\right)$, т. е. $\widetilde{u}^{0}, \widetilde{Z}_{J}^{(n)} \in W_{\gamma^{\prime}, 0}^{1}(\Omega)$ по теореме 1 . Правая часть задачи для $Z_{J}^{(\mu)}-\chi \mathscr{Z}$ равна $\sqrt{2} i\left(\widehat{a}^{0}\right)^{-1} \widetilde{u}^{0}+[\Delta, \chi] \mathscr{Z} \in\left(W_{-\gamma^{\prime}, 0}^{1}(\Omega)\right)^{*}$, и, следовательно, эта функция также принадлежит $W_{\gamma^{\prime}, 0}^{1}(\Omega)$. Теперь, взяв $\gamma^{\prime}>\gamma$, 
получаем

$$
\begin{gathered}
\left|I_{\text {com }}^{\infty}\right| \leqslant c \varepsilon^{2}\left\|v ; W_{-\gamma, 0}^{1}(\Omega)\right\|, \\
\left|I_{\text {com }}^{0}\right| \leqslant c e^{-\left(\gamma^{\prime}-\gamma\right) / \varepsilon}\left\|v ; W_{-\gamma, 0}^{1}(\Omega)\right\| \leqslant c^{\prime} \varepsilon^{2}\left\|v ; W_{-\gamma, 0}^{1}(\Omega)\right\| .
\end{gathered}
$$

Подведем итог. Правая часть формулы (5.20) принимает вид $F^{\varepsilon}(v)$, где функционал $F^{\varepsilon} \in\left(W_{-\gamma, 0}^{1}(\Omega)\right)^{*}$ имеет норму $O\left(\varepsilon^{2}\right)$, а значит, $\left\|\widehat{Z}_{J}^{\varepsilon}-\widehat{Z}_{\mathrm{Jas}}^{\varepsilon} ;\left(\widehat{\mathbf{W}}_{\gamma}^{\varepsilon 1}(\Omega)\right)^{+}\right\| \leqslant$ $c \varepsilon^{2}$, в чем и нужно было убедиться, так как норма в пространстве $\left(\widehat{\mathbf{W}}_{\gamma}^{\varepsilon 1}(\Omega)\right)^{+}$ (определяется аналогично норме в $\left.\left(\widehat{\mathbf{W}}_{\gamma}^{01}(\Omega)\right)^{+}\right)$включает в себя модули коэффициентов разложения функции. Оценки (5.9) для остатков $\widetilde{S}_{1 J}^{\varepsilon}, \ldots, \widetilde{S}_{N J}^{\varepsilon}$ и $\widetilde{S}_{J J}^{\varepsilon}$ установлены.

\section{§6. Компенсация неустойчивости простого собственного числа}

Доводы, использованные при доказательстве леммы 4 и теоремы 5, показывают, что (5.7) - собственное число задачи (1.5) в том и только в том случае, когда

$$
-1=\widehat{S}_{J J}^{\varepsilon} .
$$

Несмотря на простоту строения (см. (5.11) и (5.14), (5.17)) элемента

$$
\widehat{S}_{J J}^{\varepsilon}=-1-2 i \varepsilon\left|\widehat{a}^{0}\right|^{-2}\left(\mu-l\left(\widehat{u}^{0}, \widehat{u}^{0}\right)\right)+\widetilde{S}_{J J}^{\varepsilon},
$$

решить непосредственно уравнение (6.1) не удается из-за трудностей при контроле остатка $\widetilde{S}_{J J}^{\varepsilon}$. Выбрав $\psi \in[0, \pi)$ так, чтобы число $-e^{2 i \psi}$ не было собственным для матрицы рассеяния $s$, переформулируем уравнение (6.1) как систему

$$
\begin{gathered}
\operatorname{Im} \widehat{S}_{J J}^{\varepsilon}=0, \\
\operatorname{Re}\left(e^{i \psi}\left(\widehat{S}_{b b}^{\varepsilon}\right)^{*} \widehat{S}_{b J}^{\varepsilon}\right)=0 \in \mathbb{R}^{N} .
\end{gathered}
$$

Убедимся в том, что условия (6.3), (6.4) влекут за собой равенство (6.1) (обратное очевидно, так как $\widehat{S}_{b J}^{\varepsilon}=0$ в случае (6.1) согласно (4.10)). Из этих условий при учете взаимной ортогональности столбцов симметричной унитарной матрицы $\widehat{S}^{\varepsilon}$, а также асимптотических формул (6.2), (5.8) выводим, что

$$
\begin{aligned}
0 & =e^{i \psi}\left(\widehat{S}_{b b}^{\varepsilon}\right)^{*} \widehat{S}_{b J}^{\varepsilon}+e^{i \psi}\left(\widehat{S}_{J b}^{\varepsilon}\right)^{*} \widehat{S}_{J J}^{\varepsilon}=-e^{-i \psi}\left(\widehat{S}_{b b}^{\varepsilon}\right)^{\top} \overline{\widehat{S}_{b J}^{\varepsilon}}+e^{i \psi} \widehat{S}_{J J}^{\varepsilon} \overline{\widehat{S}_{b J}^{\varepsilon}} \\
& =-e^{-i \psi}\left(\widehat{S}_{b b}^{\varepsilon}-e^{2 i \psi} \widehat{S}_{J J}^{\varepsilon} \mathbb{I}_{N}\right) \overline{\widehat{S}_{b J}^{\varepsilon}}=-e^{-i \psi}\left(s+e^{2 i \psi} \mathbb{I}_{N}+O(\varepsilon)\right) \overline{\widehat{S}_{b J}^{\varepsilon}},
\end{aligned}
$$

причем последней в скобках стоит $(N \times N)$-матрица с элементами $O(\varepsilon)$. Итак, в случае малого $\varepsilon$ получаем равенство $\widehat{S}_{b J}^{\varepsilon}=0$, а значит, $\left|\widehat{S}_{J J}^{\varepsilon}\right|=1$ и $\widehat{S}_{J J}^{\varepsilon}=-1$ при малом $\varepsilon$ в силу условия (6.3) и асимптотики (6.2).

Решить систему (6.3), (6.4) стало несложно. Положим

$$
\mu=l\left(\widehat{u}^{0}, \widehat{u}^{0}\right)+\eta
$$

и, согласно (6.2), перепишем соотношение (6.3) следующим образом:

$$
\eta=(2 \varepsilon)^{-1}\left|\widehat{a}^{0}\right|^{2} \operatorname{Im} \widetilde{S}_{J J}^{\varepsilon} .
$$

В силу оценки (5.9) правая часть в (6.6) приобретает порядок $\varepsilon$. 
Займемся соотношением (6.4). Для того чтобы устранить влияние главного члена асимптотики столбца

$$
s^{*} \widehat{S}_{b J}^{\varepsilon}=\varepsilon 2^{1 / 2}\left(\widehat{a}^{0}\right)^{-1}\left(l\left(\widehat{u}^{0}, \zeta\right)-\mu\left(\widehat{u}^{0}, \zeta\right)_{\Omega}\right)+s^{*} \widetilde{S}_{b J}^{\varepsilon} \in \mathbb{C}^{N}
$$

(см. опять формулы $(5.14),(5.17),(5.11))$ и оставить только члены $O\left(\varepsilon\left(\left|\boldsymbol{\tau}^{\prime}\right|+|\eta|\right)\right)$ и $O\left(\varepsilon^{2}\right)$, введем условия ортогональности и нормировки

$$
\begin{gathered}
\operatorname{Re}\left(e^{i \psi}\left(l_{0}\left(\widehat{u}^{0}, \zeta_{k}\right)-l_{0}\left(\widehat{u}^{0}, \widehat{u}^{0}\right)\left(\widehat{u}^{0}, \zeta_{k}\right)_{\Omega}\right)\right)=0, \\
\operatorname{Re}\left(e ^ { i \psi } \left(\left(l_{m}\left(\widehat{u}^{0}, \zeta_{k}\right)-l_{m}\left(\widehat{u}^{0}, \widehat{u}^{0}\right)\left(\widehat{u}^{0}, \zeta_{k}\right)_{\Omega}\right)=\delta_{m, k}, \quad m, k=1, \ldots, N .\right.\right.
\end{gathered}
$$

Эти условия накладывают ограничения на возмущающие операторы (1.3) в задаче (1.2) и включают в себя собственную функцию $\widehat{u}^{0}$ и решения (3.3) исходной задачи (1.1) (в (6.7) они были собраны в строку $\left.\zeta=\left(\zeta_{1}, \ldots, \zeta_{N}\right)\right)$. Отметим, что без требований (6.8) удержать собственное число в спектре не удается (см. §7), а требования (6.9) помогают правильным образом зафиксировать параметры точной настройки $\tau_{1}, \ldots, \tau_{N}$, но выбор форм $l_{1}, \ldots, l_{N}$ достаточно произволен.

Замечание 8. Важно, что условия (6.8) влекут за собой равенства

$$
\operatorname{Im}\left(e^{i \psi}\left(l_{0}\left(\widehat{u}^{0}, \zeta_{k}\right)-l_{0}\left(\widehat{u}^{0}, \widehat{u}^{0}\right)\left(\widehat{u}^{0}, \zeta_{k}\right)_{\Omega}\right)\right)=0 \in \mathbb{R}^{N}, \quad k=1, \ldots, N,
$$

т. е. символ $\operatorname{Re}$ в (6.8) не нужен. Дело в том, что, аналогично выкладке (4.9), имеем

$$
\bar{\zeta} s=\left(\chi \overline{w_{b}^{-}}+\chi \overline{w_{b}^{+}} \bar{s}\right) s+\cdots=\chi w_{b}^{+} s+\chi w_{b}^{-}+\cdots \Longrightarrow \zeta=\bar{\zeta} s,
$$

а значит, $\operatorname{Re}\left(e^{i \psi} \bar{\zeta}\right)=0 \in \mathbb{R}^{N} \Longrightarrow \operatorname{Im}\left(e^{i \psi} \bar{\zeta}\right)=0 \in \mathbb{R}^{N}$, так как

$$
\begin{aligned}
2 i \operatorname{Im}\left(e^{i \psi} \bar{\zeta}\right) & =e^{i \psi} \bar{\zeta}-e^{-i \psi} \zeta=e^{-i \psi} \bar{\zeta}\left(e^{2 i \psi} \mathbb{I}_{N}-s\right) \\
& =e^{-i \psi} \bar{\zeta}\left(e^{2 i \psi} \mathbb{I}_{N}+s\right) m=\left(e^{i \psi} \bar{\zeta}+e^{-i \psi} \bar{\zeta} s\right) m=2 \operatorname{Re}\left(e^{i \psi} \bar{\zeta}\right) m
\end{aligned}
$$

где $m=\left(e^{2 i \psi} \mathbb{I}_{N}+s\right)^{-1}\left(e^{2 i \psi} \mathbb{I}_{N}-s\right)$ - модифицированное преобразование Кэли матрицы $s$, существующее благодаря выбору показателя $\psi$. Осталось упомянуть, что левые части формул (6.8) и (6.10) - интегралы по $\Omega$ от величин $\operatorname{Re}\left(e^{i \psi} \overline{\zeta_{k}}\right)$ и $\operatorname{Im}\left(e^{i \psi} \overline{\zeta_{k}}\right)$, умноженных на вещественную функцию.

Теперь несложными преобразованиями приводим условия (6.4) к виду

$$
\boldsymbol{\tau}^{\prime}-\eta \operatorname{Re}\left(e^{i \psi}\left(\widehat{u}^{0}, \zeta\right)_{\Omega}\right)=-2^{-1 / 2} \widehat{a}^{0} \operatorname{Re}\left(e^{i \psi}\left(\left(\widehat{S}_{b b}^{\varepsilon}-s\right)^{*} S_{b J}^{\prime}+\left(\widehat{S}_{b b}^{\varepsilon}\right)^{*} \varepsilon^{-1} \widetilde{S}_{b J}^{\varepsilon}\right)\right) .
$$

Оценки (5.8) и (5.9) показывают, что правая часть в (6.11) - величина порядка $\varepsilon$. Заменим $\eta$ выражением $(2 \varepsilon)^{-1}\left|\widehat{a}^{0}\right|^{2} \operatorname{Im} \widetilde{S}_{J J}^{\varepsilon}$ и объединим соотношения (6.6), (6.11) в систему нелинейных уравнений для вектора $\theta=\left(\tau_{1}, \ldots, \tau_{N}, \eta\right)$ :

$$
\theta=K^{\varepsilon}(\theta) \quad \text { в } \mathbb{R}^{N+1} .
$$

Согласно лемме 7 и строению правых частей равенств (6.6) и (6.11), оператор $K^{\varepsilon}$ аналитически зависит от $\varepsilon \theta$ и имеет малую норму. Следовательно, он оказывается сжимающим на шаре $\mathbb{B}_{\varepsilon \rho}^{N+1}=\{\theta:|\theta| \leqslant \varepsilon \rho\}$ с некоторым $\rho>0$. Теперь при помощи принципа сжимающих отображений находим единственное решение $\theta \in \mathbb{B}_{\varepsilon \rho}^{N+1}$ уравнения (6.12) и приходим к основному утверждению статьи.

Теорема 9. Пусть $\lambda=\lambda_{0}$ - простое собственное число задачи (1.4) из формуль (1.11), а $\widehat{u}^{0}$ - соответствующая собственная функиия, нормированная в $L_{2}(\Omega)$ и удовлетворяющая соотношению (5.1). Пусть, кроме того, 
билинейные бормы (1.6), порожденные операторами (1.3), подчинены требованиям (6.8), (6.9). Тогда существуют такие положительные величины $\varepsilon_{0}$, $c_{0}, C_{0} u \rho$, что для любого $\varepsilon \in\left(0, \varepsilon_{0}\right]$ найдется единственный вектор параметров $\left(\tau_{1}, \ldots, \tau_{N}\right) \in \mathbb{B}_{\varepsilon \rho}^{N}$, при которых задача (1.5) имеет на сегменте $\left[\lambda_{0}-c_{0}, \lambda_{0}+c_{0}\right]$ единственное собственное число $\lambda_{0}^{\varepsilon}$, подчиненное неравенству $\left|\lambda^{\varepsilon}-\lambda_{0}-\varepsilon l_{0}\left(\widehat{u}^{0}, \widehat{u}^{0}\right)\right| \leqslant C_{0} \varepsilon^{2}$.

Доказательство. Подберем $c_{0}$ так, чтобы элемент $\widehat{S}_{J J}^{0}$ расширенной матрицы рассеяния, отвечающей параметру $\lambda \in\left[\lambda_{0}-2 c_{0}, \lambda_{0}+2 c_{0}\right]$, обращался в -1 лишь при $\lambda=\lambda_{0}$. Тогда, согласно лемме 7 , уравнение (6.1) имеет не более одного решения при малых $\varepsilon$ и $\tau_{1}, \ldots, \tau_{N}$. Это решение уже было найдено из системы (6.12). Оценка погрешности в асимптотике собственного числа $\lambda^{\varepsilon}$ вытекает из (5.7) и (6.5).

\section{§7. Обсуждение результата}

Если $\lambda$ есть $\varkappa$-кратное собственное число из дискретного спектра $\sigma_{d} \subset \Upsilon_{0}$, т. е. $N=0$, то расширенная матрица рассеяния $(4.6)$, имея размер $J \times J$, совпадает со своим блоком $S_{\dagger \dagger}^{\varepsilon}$, фигурирующим в формулах (4.6) и (4.7). Повторив с незначительными изменениями выкладки из 5 , можно проверить, что $J-\varkappa$ собственных чисел расширенной матрицы рассеяния $\widehat{S}^{\varepsilon}$, отвечающей спектральному параметру (5.7), находятся вне окрестности точки -1 , а остальные в количестве $\varkappa$ штук приобретают вид

$$
\widehat{\rho}_{j}^{\varepsilon}(\mu)=-e^{i \varepsilon \eta_{j}(\mu)}+O\left(\varepsilon^{2}\right), \quad j=1, \ldots, \varkappa,
$$

где $\eta_{1}(\mu), \ldots, \eta_{\varkappa}(\mu)$ - собственные числа эрмитовой матрицы $Y(\mu)=\mu Y^{0}-Y^{1}$ с элементами $Y_{j k}^{0}=\left(u_{j}^{0}, u_{k}^{0}\right)_{\Omega}, Y_{j k}^{1}=l_{0}\left(u_{j}^{0}, u_{k}^{0}\right), j, k=1, \ldots, \varkappa$, a $\left\{u_{1}^{0}, \ldots, u_{\varkappa}^{0}\right\}$ какой-либо базис в подпространстве $\operatorname{ker}(A-\lambda)$. Поскольку собственные числа унитарной матрицы $\widehat{S}^{\varepsilon}$ располагаются на единичной окружности $\mathbb{S}^{1} \subset \mathbb{C}$, вариация параметра $\mu$ и непрерывная зависимость от него расширенной матрицы рассеяния позволяет заключить, что существуют такие точки $\mu_{1}(\varepsilon), \ldots, \mu_{\varkappa}(\varepsilon) \in \mathbb{R}$, что $\widehat{\rho}_{j}^{\varepsilon}\left(\mu_{j}(\varepsilon)\right)=-1$. В итоге, согласно теореме 5 , найдены собственные числа $\lambda_{j}^{\varepsilon}=\lambda+\varepsilon \mu_{j}(\varepsilon), j=1, \ldots, \varkappa$, оператора $A^{\varepsilon}$ (задачи (1.5)).

Если $\lambda$ - собственное число (1.11) в непрерывном спектре $\sigma_{c}$, т. е. $N \geqslant 1$, то по-прежнему собственные числа матрицы $\widehat{S}^{\varepsilon}$ допускают представления $(7.1)$ и можно найти точки $\mu_{j}(\varepsilon)$, при которых $\widehat{\rho}_{j}^{\varepsilon}\left(\mu_{j}(\varepsilon)\right)=-1$. Вместе с тем эти собственные числа могут не быть собственными числами блока $S_{\dagger \dagger}^{\varepsilon}$ и, тем самым, не порождать по теореме 5 собственные числа оператора $A^{\varepsilon}$. C другой стороны, собственные числа $\widehat{\varrho}_{j}^{\varepsilon}(\mu)$ блока $S_{\dagger \dagger}^{\varepsilon}$ также допускают представления вида $(7.1)$, однако блок, вообще говоря, не является унитарной матрицей, а значит, числа $\widehat{\varrho}_{j}^{\varepsilon}(\mu)$ могут располагаться вне единичной окружности, а пути $\mu \mapsto \widehat{\varrho}_{j}^{\varepsilon}(\mu)$ обходить точку -1 стороной. Именно в этом и состоит разница между возмущением собственных чисел на дискретном и непрерывном спектрах.

При нарушении равенств $(6.8)$ и (6.10) поправка $O(\varepsilon)$ в асимптотике $\widehat{S}_{b J}^{\varepsilon}=$ $0+\varepsilon S_{b J}^{\prime}+\cdots$ заведомо отлична от нуля, т. е. равенство (6.1) не выполнено и в $c \varepsilon^{2}$-окрестности точки $\lambda+\varepsilon l_{0}\left(\widehat{u}^{0}, \widehat{u}^{0}\right)$ собственных чисел оператора $A^{\varepsilon}$ нет. 
K сожалению, разработанные в 66 частные приемы не позволяют удержать в спектре кратное собственное число или несколько собственных чисел одновременно, и непонятно, можно ли сделать это посредством увеличения количества свободных параметров $\tau_{n}$. Вместе с тем предположение о простоте собственного числа $\Lambda_{J}$ из формулы (2.5) введено лишь для упрощения изложения. Действительно, в случае $\Lambda_{J-\kappa}<\Lambda_{J-\kappa+1}=\cdots=\Lambda_{J}<\Lambda_{J+1}, \kappa>1$, выбираем в соответствующем собственном подпространстве ортонормированный базис $\left\{U_{J-\kappa+1}, \ldots, U_{J}\right\}$ так, чтобы сохранить нужное разложение $(5.1)$ собственной функции $\widehat{u}^{0}$ задачи (1.4) (или (1.1) в дифференциальной форме). После этого все выкладки и рассуждения не нуждаются в каких-либо исправлениях.

Автор благодарен Т. А. Суслиной за полезные советы, приведшие к улучшению изложения, и за помощь в устранении опечаток в рукописи.

\section{ЛиТЕРАТУРА}

[1] М. Ш. Бирман, М. З. Соломяк, Спектралъная теория самосопряженных операторов в гилъбертовом пространстве, изд-во ЛГУ, Л., 1980.

[2] К. Фридрихс, Возмущение спектра операторов в гильбертовом пространстве, Мир, М., 1969.

[3] М. Рид, Б. Саймон, Методы современной математической физики. Т. 4. Анализ операторов, Мир, М., 1982.

[4] A. Aslanyan, L. Parnovski, D. Vassiliev, Complex resonances in acoustic waveguides, Quart J. Mech. Appl. Math., 53:3 (2000), 429-447.

[5] С. А. Назаров, Б. А. Пламеневский, Самосопряженные эллиптические задачи: операторь рассеяния и поляризачии на ребрах гранищъ, Алгебра и анализ, 6:4 (1994), 157-186.

[6] И. В. Камоцкий, С. А. Назаров, Расширенная матрища рассеяния и экспоненциально затухаюшие решения эллиптической задачи в иилиндрической области, Зап. научн. сем. ПОМИ, 264 (2000), 66-82.

[7] В. В. Грушин, О собственных значениях финитно возмущенного оператора Лапласа в бесконечных иилиндрических областях, Матем. заметки, 75:3 (2004), 360371.

[8] Р. Р. Гадыльшин, О локальных возмущениях квантовых волноводов, ТМФ, 145:3 (2005), 358-371.

[9] S. A. Nazarov, B. A. Plamenevsky, Elliptic problems in domains with piecewise smooth boundaries, Walter de Gruyter, Berlin, 1994.

[10] С. А. Назаров, Полиномиалъное свойство самосопряженных эллиптических краевых задач и алгебраическое описание их атрибутов, УМН, 54:5 (1999), 77-142.

[11] S. A. Nazarov, Properties of spectra of boundary value problems in cylindrical and quasicylindrical domain, in: Sobolev Spaces in Mathematics. II, Int. Math. Ser., vol. 9, Springer-Verlag, New York, 2009, 261-309.

[12] С. А. Назаров, Б. А. Пламеневский, Принципы излучения для самосопряженных эллиптических задач, в кн.: Пробл. матем. физики, вып. 13, изд-во ЛГУ, Л., 1991, $192-244$.

[13] С. А. Назаров, Критерий существования затухающих решений в задаче о резонаторе с иилиндрическим волноводом, Функц. анализ и его прил., 40:2 (2006), 20-32.

[14] V. Maz'ya, S. Nazarov, B. Plamenevskij, Asymptotic theory of elliptic boundary value problems in singularly perturbed domains, vol. 1, Birkhäuser, Basel, 2000. 